\title{
Etude de l'écoulement d'une suspension de fines particules en cours de cristallisation
}

\author{
N. Karouby et M. Larini \\ Laboratoire de Dynamique et Thermophysique des Fluides, \\ Laboratoire associé $n^{\circ} 72$, Université de Provence, Centre de St-Jérôme, \\ rue Poincaré, 13397 Marseille Cedex 4, France \\ (Reçu le 16 septembre 1979, révisé le 13 décembre 1979, accepté le 14 décembre 1979)
}

\begin{abstract}
Résumé. - Nous proposons dans ce travail de modéliser un écoulement diphasique constitué par de fines particules cristallisant dans un courant gazeux par les équations de l'aérothermochimie des suspensions. Nous calculons la cristallisation des particules par deux méthodes de perturbation, dont l'une est classique. Le calcul de la cristallisation est effectué en régime permanent. Nous calculons par ailleurs un écoulement simplifié particulesgaz à l'intérieur d'une conduite. Celui-ci constitue un échangeur co-courant à contact direct de sel fondu cristallisant dans un courant d'air.
\end{abstract}

\begin{abstract}
The purpose of this work is to modelise a diphasic flow constituted by the small particles solidifying in the gaseous stream by the aerothermochemistry of suspensions equations. We calculate the solidification of the particles by two methods of perturbation, one is classic. We do the assemption of stationnary state and achieve the resolution of the equation system. We modelize, also, a simplified flow, particles-gas, inside the pipe. This one is an co-courant, direct-contact heat exchanger of molten salt solidifying in an air stream.
\end{abstract}

$c_{j} \quad$ : composante $j$ de la vitesse d'une particule.

$c_{\mathrm{s}}, c_{\mathrm{L}}$ : chaleur massique de la phase solide, liquide.

$D_{\mathrm{c}} \quad$ : diamètre de la conduite.

$D_{\mathrm{p}} \quad$ : tenseur dû à la diffusion de quantité de mouvement des particules.

$e \quad$ : énergie interne massique d'une particule.

$e_{\mathrm{p}} \quad$ : énergie interne massique de la phase dispersée.

$e_{\mathrm{G}} \quad$ : énergie interne du gaz.

$F_{\mathrm{p}} \quad$ : forces agissant sur la phase dispersée.

$F_{\mathrm{p} z}$ : composante sur l'axe de $z$ de $F_{\mathrm{p}}$.

$g$ : accélération de la pesanteur.

$G_{\mathrm{p}} \quad$ : vecteur dû à la diffusion de l'énergie des particules.

$h \quad$ : coefficient de convection du gaz autour des particules.

$K \quad$ : flux de chaleur dans le gaz.

$k_{\mathrm{s}} \quad$ : coefficient de conduction de la phase solide.

$L_{\mathrm{f}} \quad$ : chaleur latente de fusion du sel.

$m(R)$ : masse d'une particule de rayon $R$.

$N_{\mathrm{B}} \quad$ : nombre de Biot $=h R / k_{\mathrm{s}}$.

$N_{\mathrm{S}} \quad:$ nombre de Stephan $=\frac{c_{\mathrm{s}}}{L_{\mathrm{f}}}\left(T_{\mathrm{f}}-T_{\infty}\right)$.

$n_{\mathrm{p}} \quad$ : nombre de particules par unité de volume.

$P \quad$ : pression du gaz.

$Q \quad$ : transfert de chaleur entre la phase dispersée et le gaz.

$R \quad$ : rayon d'une particule. $r \quad:$ distance au centre de la particule.

$R^{*} \quad$ : rayon de cristallisation.

$r^{\prime} \quad$ : distance réduite au centre de la particule.

$r^{*} \quad$ : rayon réduit de cristallisation.

$T: \quad$ : température du gaz.

$T_{\mathrm{S}} \quad$ : température dans la phase solide.

$T_{\mathrm{f}} \quad$ : température de fusion du sel.

$T_{\mathrm{w}}$ : température à la surface extérieure de la particule.

$T \quad$ : température dans le gaz loin de la particule.

$t \quad:$ temps.

$t^{\prime} \quad:$ temps réduit.

$U_{\mathbf{p}_{j}}$ : composante $j$ de la vitesse de la phase dispersée.

$v_{j} \quad$ : composante $j$ de la vitesse du gaz.

$W \quad$ : vitesse du gaz suivant l'axe de la conduite.

$W_{\mathrm{p}}$ : vitesse de la phase dispersée suivant l'axe de la conduite.

$x_{\mathbf{i}} \quad$ : variable d'espace.

$z \quad$ : cote suivant l'axe de la conduite.

$\varepsilon \quad:$ concrétisation volumique de la phase dispersée.

$\rho_{\mathrm{a}} \quad$ : masse volumique apparente de la phase dispersée.

$\rho_{\mathrm{p}} \quad$ : masse volumique réelle de la phase dispersée.

$\rho_{\mathrm{G}} \quad$ : masse volumique apparente du gaz.

$\rho_{\mathrm{GR}}$ : masse volumique réelle du gaz.

$\vec{\Gamma} \quad$ : tenseur des contraintes dans le gaz. 
$\Pi \quad$ : température réduite de la phase solide.

$\Pi_{\mathrm{w}}$ : température réduite à la surface extérieure des particules.

$\Pi_{n} \quad$ : terme d'ordre $n$ dans le développement de la température réduite (développement à partir de $\left.N_{\mathrm{B}}\right)$.

$\Pi_{n}^{\prime} \quad$ : terme d'ordre $n$ dans le développement de la température réduite (développement à partir de $N_{\mathrm{s}}$ ).

$\psi \quad$ : force agissant sur une particule.

$\psi_{\mathrm{z}} \quad$ : composante suivant l'axe de la conduite de $\psi$.

1. Introduction. - Dans ce travail nous nous proposons d'étudier le comportement d'un écoulement diphasique constitué par du chlorure de sodium pulvérisé en fines particules à l'état liquide, à une température supérieure ou égale à sa température de solidification, dans un courant d'air frais. Le sel est pulvérisé verticalement vers le haut, dans le sens de l'écoulement gazeux. En cédant de l'énergie à l'air, le chlorure de sodium se solidifie. Un tel système constitue un échangeur à co-courant à contact direct $\mathrm{NaCl}$-Air.

Pour étudier un tel échangeur il faut disposer d'un modèle d'écoulement pour les deux phases et se donner un modèle décrivant la cristallisation des particules dans l'écoulement.

En ce qui concerne celle-ci, nous avons adopté une méthode de perturbation, soit en partant du nombre de Stephan, soit du nombre de Biot. Dans les deux cas, on admet un modèle de cristallisation sphérique.

Le modèle de l'écoulement est abordé comme celui d'un écoulement diphasique.

2. Modèle d'écoulement diphasique. - Pour décrire le comportement de l'écoulement diphasique particules de $\mathrm{NaCl}$-Air, on utilise le modèle proposé par Kuentzmann [3]. Ce modèle permet de décrire le comportement des particules de sel en suspension aussi bien à l'état liquide qu'à l'état solide et en cours de cristallisation.

2.1 EQUATIONS DE LA PHASE DiSPERSÉE (PARTICULES DE CHLORURE DE SODIUM). - Dans la modélisation proposée dans la référence [3], on admet que l'on peut définir une fonction de distribution

$$
f\left(x_{i}, c_{j}, e, R, t\right) \text {. }
$$

L'utilisation de la variable $e$ est indispensable si l'on admet que l'on peut avoir un profil de température à l'intérieur de la particule. L'énergie interne massique $e$ est toujours définie à une constante près.

\subsubsection{Lorsque la particule est liquide}

$$
e-e_{\mathrm{L}}(0)=\frac{4 \Pi \rho_{\mathrm{L}} C_{\mathrm{L}}}{m(R)} \int_{0}^{R} r^{2} T(r) \mathrm{d} r
$$

$e_{\mathrm{L}}(0)=$ énergie interne massique liquide au zéro absolu.

En particulier, au début de la cristallisation si l'on admet que la particule liquide a une température uniforme et égale à la température de changement de phase

$$
e\left(T_{\mathrm{f}}\right)-e_{\mathrm{L}}(0)=C_{\mathrm{L}} T_{\mathrm{f}}
$$

2.1.2 Lorsque la particule est solide (après la fin de la cristallisation)

$$
e-e_{\mathrm{s}}(0)=\frac{4 \Pi \rho_{\mathrm{S}} C_{\mathrm{S}}}{m(R)} \int_{0}^{R} r^{2} T(r) \mathrm{d} r
$$

$e_{\mathrm{s}}(0)=$ énergie interne massique solide au zéro absolu.

2.1.3 En cours de cristallisation. - Si l'on admet par exemple que la particule au début de la cristallisation est à une température uniforme égale à la température de fusion :

$$
e=e\left(T_{\mathrm{f}}\right)-\mathcal{F} \Rightarrow e-e_{\mathrm{L}}(0)=C_{\mathrm{L}} T_{\mathrm{f}}-\mathcal{F}
$$

où $\mathcal{F}$ est la quantité de chaleur par unité de masse cédée au milieu extérieur depuis le début de la cristallisation.

La fonction de distribution définie est telle que :

$$
\mathrm{d} n_{\mathrm{p}}=f\left(x_{i}, c_{j}, e, R, t\right) d_{3} x_{i} d_{3} \mathrm{~d} e \mathrm{~d} R
$$

représente le nombre probable de particules se trouvant à l'instant $t$ dans un élément de volume de l'espace des phases généralisé, autour du point de coordonnées $x_{i}, c_{j}, e, R$.

L'existence de cette fonction de distribution permet de définir des grandeurs macroscopiques de la phase dispersée

$$
\begin{aligned}
n_{\mathrm{p}}\left(x_{i}, t\right) & =\int f d_{3} c_{j} \mathrm{~d} e \mathrm{~d} R \\
\rho_{\mathrm{a}}\left(x_{i}, t\right) & =\int m(R) f d_{3} c_{j} \mathrm{~d} e \mathrm{~d} R \\
U_{\mathrm{p}_{k}}\left(x_{i}, t\right) & =\frac{1}{\rho_{\mathrm{a}}} \int m(R) c_{k} f d_{3} c_{j} \mathrm{~d} e \mathrm{~d} R \\
e_{\mathrm{p}}\left(x_{i}, t\right) & =\frac{1}{\rho_{\mathrm{a}}} \int m(R) e f d_{3} c_{j} \mathrm{~d} e \mathrm{~d} R
\end{aligned}
$$

On peut par ailleurs définir une concentration volumique de la phase dispersée

$$
\varepsilon\left(x_{i}, t\right)=\frac{\rho_{\mathrm{a}}}{\rho_{\mathrm{p}}} .
$$

La fonction de distribution obéit à une équation de bilan du type Liouville-Boltzman

$$
\frac{\partial f}{\partial t}+\sum_{i} \frac{\partial\left(f \dot{q}_{i}\right)}{\partial q_{i}}=J\left(q_{i}, t\right)
$$


où les $q_{i}$ sont les coordonnées généralisées de l'espace des phases.

$\mathrm{Si}$ l'on néglige les effets dus aux collisions entre les particules, à leur coalescence, à la nucléation, à leur éclatement $J\left(q_{i}, t\right)=0$.

Si par ailleurs, on admet qu'il n'y a pas de transfert de masse entre la phase dispersée et le gaz, par intégration de (6) multipliée successivement par $m(R)$, $m(R) c_{j}, m(R) e$ on obtient, compte tenu des équations (1) à (6), après quelques transformations classiques :

- Equation de bilan de masse :

$$
\frac{\partial \rho_{\mathrm{a}}}{\partial t}+\operatorname{Div} \rho_{\mathrm{a}} \mathbf{U}_{\mathrm{p}}=0
$$

- Equation de bilan de quantité de mouvement :

$$
\begin{gathered}
\rho_{\mathrm{a}} \frac{\partial \mathbf{U}_{\mathrm{p}}}{\partial t}+\rho_{\mathrm{a}} \mathbf{U}_{\mathrm{p}} \cdot \operatorname{grad} \mathbf{U}_{\mathrm{p}}=\rho_{\mathrm{a}} \mathbf{g}+\mathbf{F}_{\mathrm{p}}+\operatorname{Div} \mathbf{D}_{\mathrm{p}} \\
\mathbf{F}_{\mathrm{p}}=\int \psi f d_{3} c_{j} \mathrm{~d} e \mathrm{~d} R
\end{gathered}
$$

où $\psi$ représente la force exercée par le gaz sur une particule.

$\mathbf{D}_{\mathrm{p}}$ est un tenseur symétrique d'ordre deux dû à la diffusion des particules dans leur mouvement moyen.

- Equation de bilan de l'énergie interne

$$
\begin{gathered}
\rho_{\mathrm{a}} \frac{\partial e_{\mathrm{p}}}{\partial t}+\rho_{\mathrm{a}} \mathbf{U}_{\mathrm{p}} \cdot \operatorname{grad} \mathbf{e}_{\mathrm{p}}=-Q-\operatorname{div} \mathbf{G}_{\mathrm{p}} \\
Q \doteq \int q f d_{3} c_{j} \mathrm{~d} e \mathrm{~d} R
\end{gathered}
$$

où $q$ est le flux thermique cédé au gaz par une particule.

$\mathbf{G}_{\mathrm{p}}$ est un vecteur dû à la diffusion des particules dans leur mouvement moyen.

2.2 Equations DE L'ÉCOUlement DE LA PHASE CONTINUE (AIR). - Les équations obtenues dans la référence [3] sont :

- Equation de bilan de masse

$$
\frac{\partial \rho_{\mathrm{G}}}{\partial t}+\operatorname{Div} \rho_{\mathrm{G}} \mathbf{v}=0
$$

Si $\rho_{\mathrm{GR}}$ est la masse volumique réelle du gaz

$$
\rho_{\mathrm{G}}=(1-\varepsilon) \rho_{\mathrm{GR}} \text {. }
$$

- Equation de bilan de quantité de mouvement

$$
\rho_{\mathrm{G}} \frac{\mathrm{d} \mathbf{v}}{\mathrm{d} t}=\operatorname{div} \overrightarrow{\boldsymbol{\Gamma}}+\rho_{\mathrm{G}} \mathbf{g}-\mathbf{F}_{\mathrm{p}}
$$

a vec

$$
\frac{\mathrm{d}}{\partial t}=\frac{\partial}{\partial t}+v_{i} \frac{\partial}{\partial x_{i}}
$$

- Equation de bilan d'énergie

$$
\begin{aligned}
\rho_{\mathrm{G}} \frac{\mathrm{d}}{\mathrm{d} t}\left(e_{\mathrm{G}}+\frac{v^{2}}{2}\right)=\rho_{\mathrm{G}} \mathbf{g} \cdot \mathbf{v}+\operatorname{div}(\overrightarrow{\boldsymbol{\Gamma}} \cdot \mathbf{v})+ \\
+\operatorname{div} \mathbf{K}+Q-\mathbf{U}_{\mathbf{p}} \cdot \mathbf{F}_{\mathbf{p}} .
\end{aligned}
$$

3. Cristallisation d'une particule dans un milieu infini à température constante. - On étudie la cristallisation avec des hypothèses restrictives classiques $[4,5,6,7,8]$. On admet que la cristallisation s'effectue avec une symétrie sphérique, que la masse volumique du liquide est égale à la masse volumique du solide. On suppose que la température de la phase liquide reste égale à la température de changement de phase.

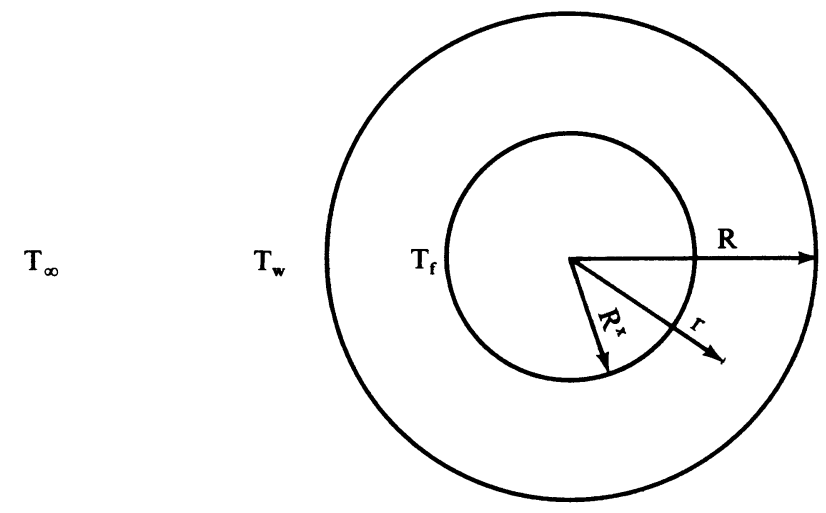

Diverses méthodes peuvent être utilisées pour résoudre le problème de la cristallisation vers l'intérieur pour une particule sphérique. On peut imaginer un calcul dérivé de la méthode proposée par Goodman [7] où l'on suppose une forme particulière du profil de température dans la croûte solide. On peut également mettre en œuvre un calcul numérique complet couplant l'évolution de la température du gaz avec la cristallisation [10].

Nous avons préféré utiliser une méthode de perturbation à partir du nombre de Stephan d'une part, et du nombre de Biot d'autre part, afin de prendre en compte des paramètres physiques, ce que ne permet pas la méthode proposée par Goodman. Les deux calculs sont développés pour une température à l'infini constante. Leur utilisation dans un processus de cristallisation où la température à l'infini varie en fonction de la cote constitue une approximation. Toutefois, celle-ci peut être considérée du même type que celle qui nous autorise à utiliser des formules de corrélation, établies en régime stationnaire, pour exprimer soit la force agissant sur une particule soit le coefficient de convection.

Le phénomène de cristallisation est régi par :

- Equation de bilan thermique dans la croûte solide,

$$
\frac{\partial T_{\mathrm{S}}}{\partial t}=\frac{k_{\mathrm{S}}}{\rho_{\mathrm{p}} C_{\mathrm{S}}} \frac{1}{r^{\prime}} \frac{\partial^{2} r T_{\mathrm{S}}}{\partial r^{2}} .
$$


- Deux conditions de température

$$
\begin{aligned}
T_{\mathrm{S}}(R) & =T_{\mathrm{W}} & & \text { inconnue } \\
T_{\mathrm{S}}\left(R^{*}\right) & =T_{\mathrm{f}} & & \text { fixée } .
\end{aligned}
$$

- Deux conditions de flux

$$
k_{\mathrm{S}}\left(\frac{\hat{\partial} T_{\mathrm{S}}}{\partial r}\right)_{\mathrm{R}}=-h\left(T_{\mathrm{W}}-T_{\infty}\right)
$$

à la périphérie externe

$$
\rho_{\mathrm{p}} L_{\mathrm{f}} \frac{\mathrm{d} R^{*}}{\mathrm{~d} t}=k_{\mathrm{S}}\left(\frac{\partial T_{\mathrm{S}}}{\partial r}\right)_{R^{*}}
$$

à l'interface liquide solide.

Si l'on définit les paramètres adimensionnels suivants

$$
\begin{aligned}
\Pi & =\frac{T_{\mathrm{S}}-T_{\mathrm{f}}}{T_{\infty}-T_{\mathrm{f}}} & t^{\prime} & =\frac{k_{\mathrm{S}}\left(T_{\mathrm{f}}-T_{\infty}\right)}{\rho_{\mathrm{p}} L_{\mathrm{f}} R^{2}} t \\
r^{*} & =\frac{R^{*}}{R} & N_{\mathrm{S}} & =\frac{C_{\mathrm{S}}}{L_{\mathrm{f}}}\left(T_{\mathrm{f}}-T_{\infty}\right) \\
r^{\prime} & =\frac{r}{R} & N_{\mathrm{B}} & =\frac{h R}{k_{\mathrm{S}}},
\end{aligned}
$$

l'équation (15) devient

$$
\frac{\mathrm{d} r^{*}}{\mathrm{~d} t^{\prime}}=-\left(\frac{\partial \Pi}{\partial r^{\prime}}\right)_{r^{*}}
$$

Si l'on écrit l'équation (13) sous forme adimensionnelle et si l'on élimine $t^{\prime}$ à l'aide de l'équation (16), il vient

$$
-N_{\mathrm{S}}\left(\frac{\partial \Pi}{\partial r^{\prime}}\right)_{r^{*}} \times\left(\frac{\partial \Pi}{\partial r^{*}}\right)=\frac{1}{r} \frac{\partial^{2} r^{\prime} \Pi}{\partial r^{\prime 2}}
$$

Les conditions aux limites de cette équation sont

$$
\begin{aligned}
\Pi(1) & =\Pi_{\mathrm{w}}=\frac{T_{\mathrm{w}}-T_{\mathrm{f}}}{T_{\infty}-T_{\mathrm{f}}} \\
\Pi\left(r^{*}\right) & =0
\end{aligned}
$$

enfin, la condition de flux à la périphérie externe (14) devient

$$
\left(\frac{\partial \Pi}{\Pi r^{\prime}}\right)_{1}=-N_{\mathrm{B}}\left[\Pi_{\mathrm{w}}-1\right]
$$

Les équations (17) et (18) montrent que la température réduite peut être considérée comme une fonction du rayon de cristallisation, de la position dans le solide, du nombre de Stephan $\left(N_{\mathrm{S}}\right)$ et du nombre de Biot $\left(N_{\mathrm{B}}\right)$.

Si $N_{\mathrm{B}}$ ou $N_{\mathrm{S}}$ sont petits, on peut développer la température réduite à partir de ces petits paramètres

ou

$$
\Pi\left(r^{*}, r^{\prime}, N_{\mathrm{B}}, N_{\mathrm{S}}\right)=\sum_{i} N_{\mathrm{S}}^{i} \Pi_{i}^{\prime}\left(r^{*}, r^{\prime}, N_{\mathrm{B}}\right)
$$

$$
\Pi\left(r^{*}, r^{\prime}, N_{\mathrm{B}}, N_{\mathrm{S}}\right)=\sum_{i} N_{\mathrm{B}}^{i} \Pi_{i}\left(r^{*}, r^{\prime}, N_{\mathrm{S}}\right)
$$

3.1 DÉVElopPement A PARTIR DU NOMBRE DE STEPHAN. - Un tel développement a déjà été utilisé [4, 8] pour étudier la cristallisation vers l'intérieur d'une particule sphérique soit pour un flux pariétal constant soit pour une température à l'infini constante. Nous ne développons pas ici ce type de solution qui est désormais classique. Les résultats obtenus par les différents auteurs montrent que les profils de température ou l'évolution des rayons de cristallisation peuvent diverger quand $r^{*} \rightarrow 0$. Cette divergence diffère suivant l'ordre de développement et se manifeste d'autant plus tard que $N_{\mathrm{S}}$ et $N_{\mathrm{B}}$ sont plus petits. Dans le présent travail nous utilisons les solutions de ce développement à l'ordre 0 et à l'ordre 1 .

3. 2 DÉVELOPPEMENT A PARTIR DU NOMBRE DE BIOT. - L'échange convectif entre la particule et le milieu extérieur étant un paramètre essentiel dans l'évolution de la cristallisation il nous a semblé intéressant de chercher une solution construite sur le développement de la température réduite à partir du nombre de Biot (20).

Si le nombre de Biot tend vers zéro, cela correspond à une barrière thermique infinie. Tout se passe comme si la particule est isolée du milieu extérieur. L'intérieur de la particule est alors en équilibre thermique, c'est-à-dire à la température de changement de phase $T_{\mathrm{f}}$.

Dans ce cas

$$
\Pi_{0}\left(r^{*}, r^{\prime}, N_{\mathrm{S}}\right)=0
$$

et si l'on se limite à l'ordre zéro l'équation (16) conduit à une solution triviale $r^{*}$ indéterminée.

L'équation (17) s'écrit alors pour le développement (20)

$-N_{\mathrm{S}} \sum_{n} \sum_{m} N_{\mathrm{B}}^{(n+m)}\left(\frac{\partial \Pi_{n}}{\partial r^{\prime}}\right)_{r^{*}} \times \frac{\partial \Pi_{m}}{\partial r^{*}}=\frac{1}{r^{\prime}} \sum_{i} N_{\mathrm{B}}^{i} \frac{\partial^{2} r^{\prime} \Pi_{i}}{\partial r^{\prime 2}}$

alors que l'équation (18) devient

$$
\sum_{n} N_{\mathrm{B}}^{n}\left(\frac{\partial \Pi_{n}}{\partial r^{\prime}}\right)_{1}=N_{\mathrm{B}}-\sum_{i} N_{\mathrm{B}}^{(i+1)} \Pi_{\mathrm{W} i}
$$

La condition à la limite $r^{\prime}=r^{*}$ est :

$$
\Pi_{n}\left(r^{*}\right)=0 \quad \forall n
$$

\subsubsection{Solution de rang 1}

$$
\begin{aligned}
& \frac{\partial^{2} r^{\prime} \Pi_{1}}{\partial r^{\prime 2}}=0 \\
& \left(\frac{\partial \Pi_{1}}{\partial r^{\prime}}\right)_{1}=1 .
\end{aligned}
$$

(21.1) et (22.1) conduisent à la solution

$$
\Pi_{1}=\frac{1}{r^{*}}-\frac{1}{r^{\prime}} \text {. }
$$


L'équation (16) s'intègre analytiquement, on obtient

$$
r^{*}=\left(1-3 N_{\mathrm{B}} t^{\prime}\right)^{1 / 3} .
$$

\subsubsection{Solution de rang 2}

$$
\begin{gathered}
-N_{\mathrm{S}}\left(\frac{\partial \Pi_{1}}{\partial r^{\prime}}\right)_{r^{*}} \frac{\partial \Pi_{1}}{\partial r^{*}}=\frac{1}{r^{\prime}} \frac{\partial^{2} r^{\prime} \Pi_{2}}{\partial r^{\prime 2}} \\
\left(\frac{\partial \Pi_{2}}{\partial r^{\prime}}\right)_{1}=-\Pi_{\mathrm{w}_{1}} .
\end{gathered}
$$

La résolution de $(21.2)$ et $(22.2)$ conduit à

$$
\begin{aligned}
\Pi_{2}= & \frac{N_{\mathrm{s}}}{6} \frac{1}{r^{* 4}} r^{2}+\left(\frac{N_{\mathrm{s}}}{3} \frac{1}{r^{* 4}}+\frac{1}{r^{*}}-1\right) \times \\
& \times \frac{1}{r^{\prime}}-\frac{N_{\mathrm{s}}}{6} \frac{1}{r^{* 4}}\left(r^{* 2}+\frac{2}{r^{*}}\right)-\frac{1}{r^{*}}\left(\frac{1}{r^{*}}-1\right) .
\end{aligned}
$$

Si l'on porte dans l'équation (16) la température réduite à l'ordre 2

$$
\Pi=N_{\mathrm{B}} \Pi_{1}+N_{\mathrm{B}}^{2} \Pi_{2} .
$$

On obtient une équation d'évolution du rayon de cristallisation à l'ordre 2 .

Sur la figure 1 on a représenté l'évolution du rayon de cristallisation en fonction du temps adimensionnel pour un nombre de Biot de 0,06 et différentes valeurs du nombre de Stephan. La solution d'ordre 1 ne dépend pas du nombre de Stephan. La solution d'ordre deux présente une asymptote horizontale dont la position dépend du nombre de Stephan. Cette asymptote est une singularité mathématique qui limite l'utilisation de ce type de développement. On sera donc conduit à arrêter le calcul avant la fin de la cristallisation.

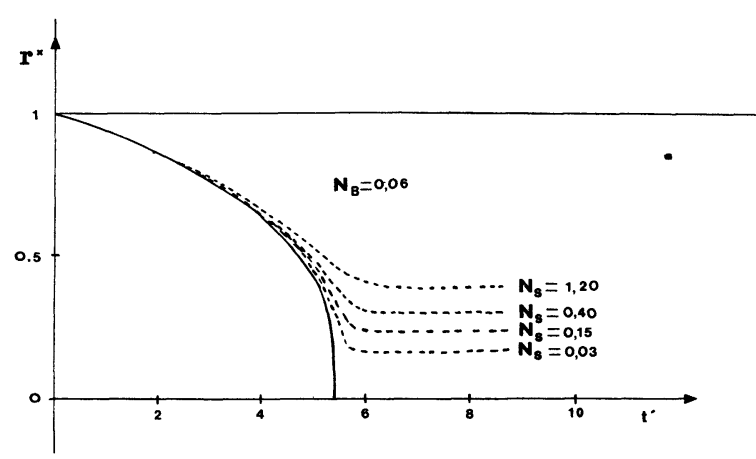

Fig. 1. - Evolution du rayon de cristallisation d'une particule dans un milieu infini en fonction du temps réduit pour le développement à partir de $N_{\mathrm{B}}$ : - Sol d'ordre 1; ------ Sol d'ordre 2.

[The evolution of the solidification radius for one particle in the boundless middle acting the recess time for $N_{\mathrm{B}}$ development.]

4. Calcul d'un écoulement simplifié. - On étudie un écoulement diphasique constitué de particules de sel fondu entraînées dans un courant d'air vertical de bas en haut. La conduite est cylindrique.
Les effets de viscosité et de conduction dans le gaz ne sont pris en compte qu'au niveau des interfaces sel-gaz. Les interactions de l'écoulement avec la paroi sont négligées. On suppose que les grandeurs macroscopiques caractérisant l'écoulement de la suspension et du gaz ne dépendent que de la cote et que par ailleurs à une cote $z$ donnée les grandeurs ne dépendent pas du temps.

Toutes les particules de sel ont même dimension et sont pulvérisées à l'état liquide à la cote $z=0$ avec la même vitesse et à la température de changement de phase. De ce fait, la cristallisation des particules commence dès la cote $z=0$.

Compte tenu de ces hypothèses, les équations (7), ..., (12) deviennent :

Phase dispersée

$$
\begin{aligned}
& \frac{\mathrm{d}}{\mathrm{d} z}\left(\rho_{\mathrm{p}} \varepsilon W_{\mathrm{p}}\right)=0 \\
& \varepsilon \rho_{\mathrm{p}} W_{\mathrm{p}} \frac{\mathrm{d} W_{\mathrm{p}}}{\mathrm{d} z}=F_{\mathrm{p}_{\mathrm{z}}}-\varepsilon \rho_{\mathrm{p}} g \\
& \varepsilon \rho_{\mathrm{p}} W_{\mathrm{p}} \frac{\mathrm{d}}{\mathrm{d} z} e_{\mathrm{p}}=-Q .
\end{aligned}
$$

Phase continue

$$
\begin{gathered}
\frac{\mathrm{d}}{\mathrm{d} z}\left((1-\varepsilon) \rho_{\mathrm{GR}} W\right)=0 \\
(1-\varepsilon) \rho_{\mathrm{GR}} W \frac{\mathrm{d} W}{\mathrm{~d} z}+\frac{\mathrm{d} P}{\mathrm{~d} z}=-\rho_{\mathrm{GR}}(1-\varepsilon) g-F_{\mathrm{p}_{z}}
\end{gathered}
$$

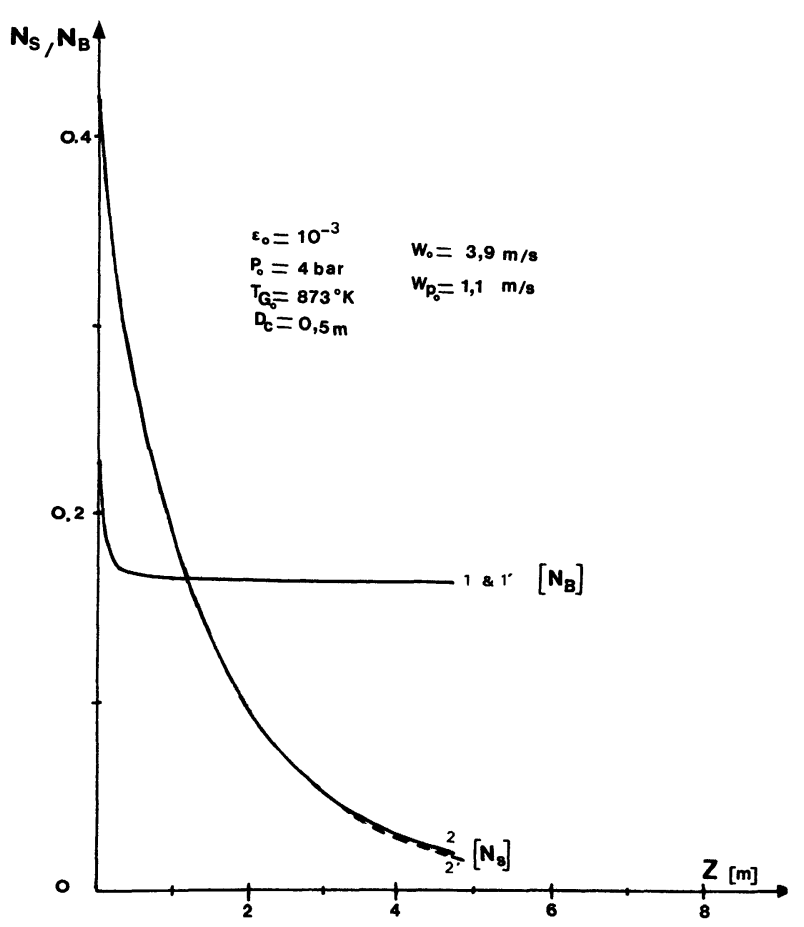

Fig. 2. - Evolution de $N_{\mathrm{B}}$ et de $N_{\mathrm{S}}$ le long de la conduite : 1 et 2 développement à partir de $N_{\mathrm{S}} ; 1^{\prime}$ et $2^{\prime}$ développement à partir de $N_{\mathrm{B}}$.

[The evolution of $N_{\mathrm{B}}$ and $N_{\mathrm{S}}$ along the pipe.] 


$$
\begin{gathered}
(1-\varepsilon) \rho_{\mathrm{GR}} W \frac{\mathrm{d}}{\mathrm{d} z}\left(e_{\mathrm{G}}+\frac{W^{2}}{2}\right)= \\
=-\rho_{\mathrm{GR}}(1-\varepsilon) g W-P \frac{\mathrm{d} W}{\mathrm{~d} z}-W \frac{\mathrm{d} P}{\mathrm{~d} z}+Q \\
P=\rho_{\mathrm{GR}} R T .
\end{gathered}
$$

Les particules ayant même rayon et étant injectées à la même vitesse, le tenseur $\mathbf{D}_{p}(8)$ et le vecteur $\mathbf{G}_{\mathrm{p}}(9)$ dus à la diffusion des particules sont nuls.

D'autre part $F_{\mathrm{p}_{z}}$ et $Q$ s'expriment alors simplement

ou

$$
\begin{aligned}
F_{\mathbf{p}_{\mathrm{z}}} & =n_{\mathrm{p}} \psi_{z} \\
Q & =n_{\mathrm{p}} q
\end{aligned}
$$

$$
q=4 \Pi R^{2} h\left(T_{\mathrm{w}}-T_{\infty}\right) .
$$

$T_{\mathrm{W}}$ résulte de la résolution couplée des équations régissant la cristallisation et l'écoulement.

$T_{\infty}$ est prise égale à la température du gaz à la cote $z$ considérée.

$\psi$ et $h$ sont exprimés à partir des formules de corrélation suivantes

$$
\begin{gathered}
C_{\mathrm{D}}=\frac{24}{R_{\mathrm{e}}}\left(1+\frac{1}{16} R_{\mathrm{e}}\right) \\
\frac{h}{C_{\mathrm{p}}\left|W-W_{\mathrm{p}}\right| \rho_{\mathrm{GR}}}=\frac{2,2}{R_{\mathrm{e}}}+\frac{0,48}{R_{\mathrm{e}}^{1 / 2}} .
\end{gathered}
$$

Nous avons résolu l'ensemble des équations régissant l'écoulement diphasique compte tenu de la cristallisation des particules de sel par une méthode numérique aux différences finies explicite du deuxième ordre (Méthode d'Euler modifiée).

Dans la section $(z)=0$ on se donne la concentration volumique du sel, la pression, la vitesse du gaz, la vitesse des particules et la température du gaz. Nous rappelons que les particules de sel dans la section $z=0$ sont à l'état liquide et à la température de fusion.

La figure 2 représente l'évolution des nombres de Biot et de Stephan le long de la conduite pour les écoulements calculés à partir des modèles de cristallisation Biot ordre deux et Stephan ordre 1. Les valeurs obtenues pour ces conditions montrent que $N_{\mathrm{B}}$ et $N_{\mathrm{S}}$ sont toujours très inférieures à un. Ce qui tend à valider à postériori les méthodes de perturbation pour les cas étudiés. On remarque que le nombre de Stephan devient petit lorsque le rayon de cristallisation devient petit, ce qui prolonge le domaine de validité de la solution.

Sur la figure 3 , on montre l'évolution du rayon de cristallisation le long de l'écoulement.

Chaque courbe (a), (b), (a'), ( $\left.b^{\prime}\right)$ correspond à un modèle de cristallisation différent.

(a) Développement en $N_{\mathrm{B}}$ à l'ordre un.

(b) Développement en $N_{\mathrm{B}}$ à l'ordre deux.

(a) Développement en $N_{\mathrm{S}}$ à l'ordre zéro.

(b) Développement en $N_{\mathrm{S}}$ à l'ordre un.

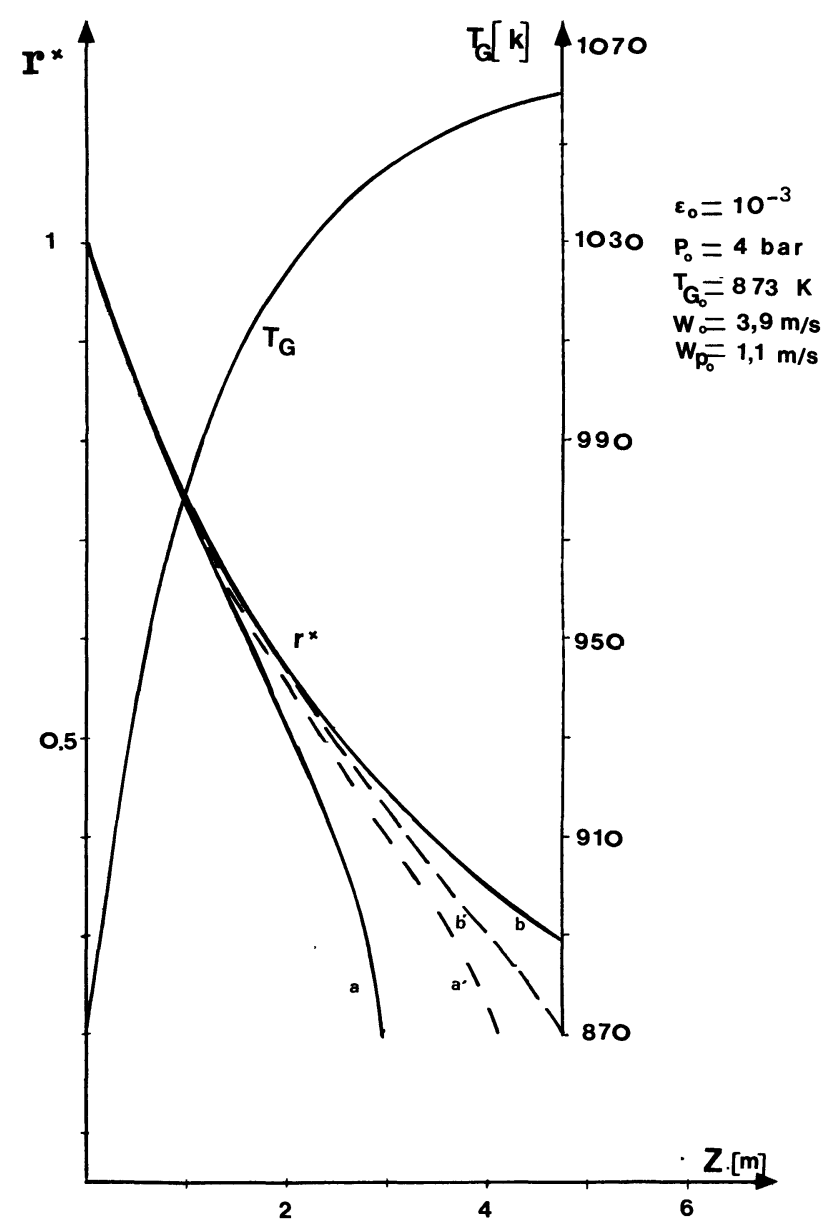

Fig. 3. - Evolution de la température du gaz et du rayon de cristallisation le long de la conduite :- développement en $N_{\mathrm{B}}$ : $a$ ) ordre $1, b$ ) ordre $2 ;-\cdots-$ développement en $N_{\mathrm{S}}: a$ ) ordre 0 , b) ordre 1 .

[The evolution of the gas temperature and cristallization radius along the pipe.]

Les courbes (b) et ( $\left.b^{\prime}\right)$ qui correspondent au développement d'ordre supérieur sont en bon accord jusqu'à des valeurs du rayon de cristallisation réduit de 0,35 . Pour les valeurs inférieures, la courbe (b) semble diverger. On retrouve en écoulement la même singularité que pour la figure 1 . Bien que le nombre de Stéphan soit très petit la singularité sur le rayon se manifeste dans ce cas avant que $99 \%$ de la masse soit cristallisée (limite que l'on se donne pour le calcul).

Sur la figure 3 l'évolution de la température du gaz au cours de l'écoulement a été représentée pour les modèles de cristallisation relatifs aux courbes (b) et $\left(b^{\prime}\right)$. L'accord entre les deux modèles est meilleur pour l'évolution de la température du gaz que pour l'évolution du rayon de cristallisation. Ceci s'explique par le fait que l'énergie libérée par la cristallisation est en fait proportionnelle au volume cristallisé et non au rayon.

Sur la figure 4 on donne pour différents rayons de particules l'évolution du cube des rayons de cristal- 


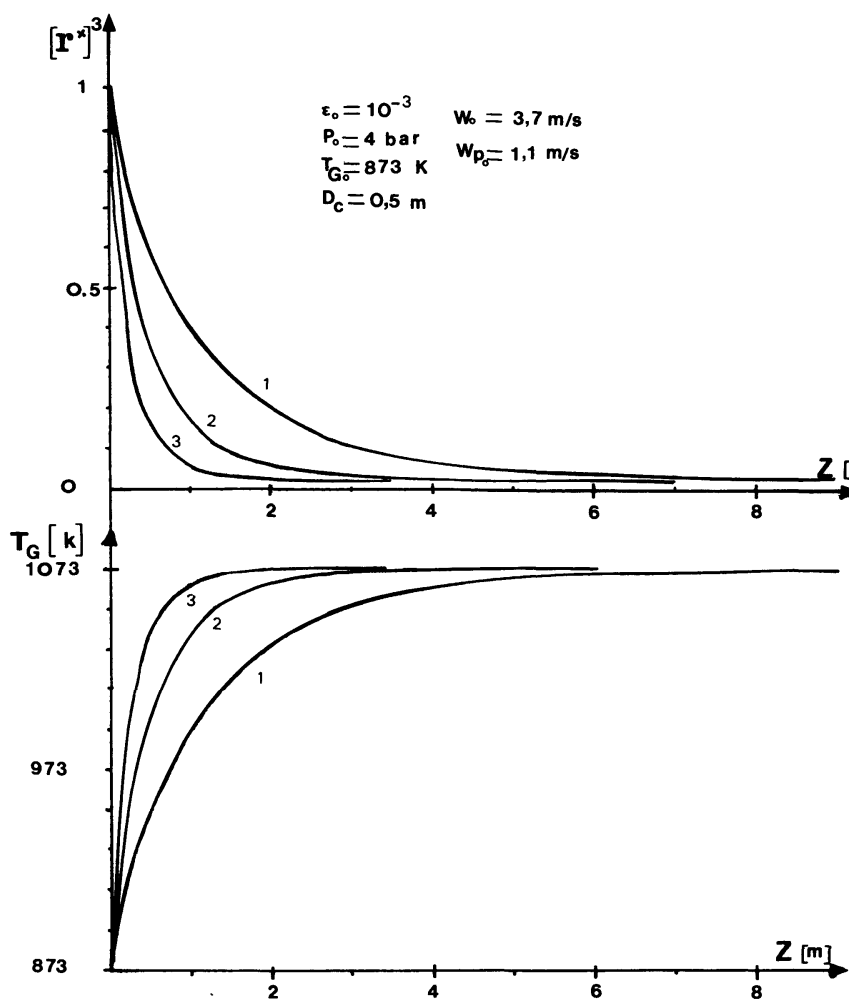

Fig. 4. - Evolution de la température du gaz et du cube de rayon de cristallisation le long de la conduite. $1: R=125 \mu ; 2: R=75 \mu$; $3: R=50 \mu$.

[The evolution of the gas temperature and the cubic cristallization radius along the pipe.]

lisation et celle de la température du gaz en fonction de la cote pour le développement en $N_{\mathrm{B}}$ à l'ordre deux. On n'a pas représenté le cas correspondant au développement en $N_{\mathrm{S}}$ à l'ordre un, pour ne pas surcharger les figures. Les courbes pour les deux types de développement sont en parfait accord.

Sur cet exemple, le rapport débit de sel, débit de gaz est tel que le gaz parvient à atteindre la température de changement de phase du chlorure de sodium alors que celui-ci est cristallisé à environ $99 \%$.

Ce cas constitue un fonctionnement idéal de l'échangeur à contact direct à co-courant. La figure 4 montre aussi l'influence du rayon des particules de sels sur la longueur de l'échangeur.

Sur la figure 5 on montre pour les mêmes conditions que sur la figure 4 l'évolution de la température de surface des particules au cours de l'écoulement.

Dans le cas des figures 4 et 5 les nombres de Biot et de Stephan restent toujours très inférieurs à un.

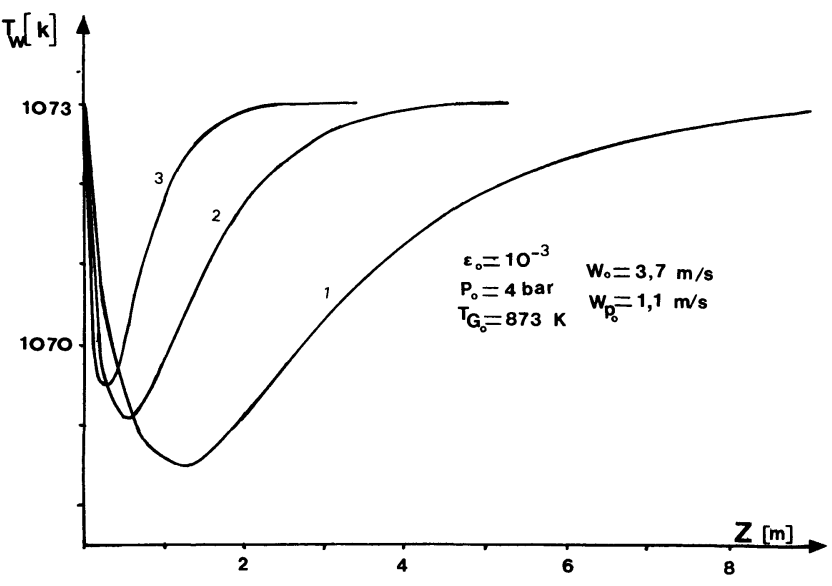

Fig. 5. - Evolution de la température de surface des particules le long de la conduite. $1: R=125 \mu ; 2: R=75 \mu ; 3: R=50 \mu$.

[The evolution of the wall particle temperature along the pipe.]

5. Conclusion. - Les deux modèles de cristallisation utilisés sont en bon accord surtout en ce qui concerne l'évolution de la température du gaz au cours de l'écoulement. Bien que pouvant présenter des singularités, de tels modèles semblent pouvoir être utilisés si $N_{\mathrm{S}}$ et $N_{\mathrm{B}}$ sont petits à condition d'arrêter le calcul alors qu'il reste encore un à deux pour cent en masse à cristalliser.

Dans la réalité la masse volumique du solide est plus grande que celle du liquide. Il en résulte qu'en fin de cristallisation la particule présente une cavité. Ce fait est confirmé par des expériences réalisées au laboratoire de l'Ecole Centrale avec du $\mathrm{NaCl}$ et au L.T.D.F. de Marseille avec de l'hitec. Ce fait semble nous autoriser à arrêter dans notre modèle le calcul de la cristallisation avant que la phase solide n'ait atteint le centre de la particule. Toutefois, ultérieurement nous développerons des modèles de cristallisation prenant en compte la différence de masse volumique entre la phase liquide et la phase solide.

Les résultats présentés sur la courbe III sont très optimistes. Dans la réalité on ne pourra jamais obtenir un tel fonctionnement idéal car la répartition dans l'espace des particules n'est pas uniforme. Ceci doit nous inciter à compléter la modélisation et à tenir compte en particulier de la distribution en taille des particules et de l'aspect bidimensionnel de la répartition des particules dans l'espace. Par ailleurs le développement d'expériences en parallèle afin de contrôler la validité des modèles nous semble indispensable. 


\section{Bibliographie}

[1] FAVRe, O., Contribution à l'étude d'un échangeur dynamique à contact direct par injection d'un sel fondu dans un fluide caloporteur (Thèse de Doctorat de spécialité : Université de Provence 1978).

[2] Pantaloni, J., Larini, M., Guenoche, H., Desaulty, M., Boyer, J., Petrt, B., Huetz, J., Aperçu sur les possibilités de stockage thermique à haute température. Rev. Gén. Therm. (août 1979).

[3] Kuentzmann, P., Aerothermo-chimie des suspensions (Mémoire de Sciences physiques, Gauthier-Villars) 1973.

[4] Pedroso, R. I., Demoto, G. A., Perturbation solutions for spherical solidification of saturated liquids ( $J$. Heat Transfer (1973) 42-46).

[5] Riley, D. S., Smith, F. T., Poots, G., The inward solidification of spheres and circular cylinders (Int. heat and mass transfer 17 (1974) 1507-1516).
[6] Vijay K Dhir, Kin Wong and Kastenberg, W. E., Solidification of a sphere with internal heat generation and convective cooling at the surface. Technical note Nuclear and Sciences Engineering, p. 350-356.

[7] Goodman, The heat balance integral and its applications to problems invalring a change of phase (Trans. ASME 80 (1958) 335-342).

[8] Ching-Lan Huang and Yen-Ping Shit, A perturbation method for spherical and cylindrical solidification (Chem. Eng. Sci. 30 (1975) 897-906).

[9] Barrere, M. et Prud'homme, R., Aérothermochimie des écoulements à deux phases (chimie et industrie, génie chimique 102 (1969)).

[10] Desaulty, M., Etude d'un échangeur à contre-courant gazgouttes de chlorure de sodium en cours de cristallisation (à paraître). 